\title{
La traction animale à l'Office du Niger au Mali : du colonat au désengagement de l'Etat
}

\author{
H. Kassambara ${ }^{1}$ P. Kleene ${ }^{2}$
}

\begin{abstract}
Mots-clés
Energie animale - Politique agricole Delta - Fleuve Niger - Mali.
\end{abstract}

\begin{abstract}
Résumé
L'Office du Niger a commencé son exploitation au début des années 1930 par la traction bovine. La grande mécanisation, introduite à partir de 1949, s'est soldée par un échec dix ans plus tard. Depuis, la traction animale est redevenue la principale source d'énergie pour le travail du sol et le transport. Jusqu'au début des années 1980, les systèmes de production étaient basés sur des techniques extensives, conduisant à des rendements moyens en paddy qui ne dépassaient que rarement 2 t/ha. A partir de 1982, une nouvelle dynamique dans les activités de l'Office du Niger s'est instaurée. La traction animale s'est fortement développée, grâce à de nouvelles facilités de crédit, du matériel mieux adapté fabriqué sur place et des services vétérinaires adéquats. Les rendements moyens en paddy sont passés à 4-6 t/ha, en fonction du type d'aménagement. La restructuration de l'Office du Niger, intervenue en 1994, a vu la suppression de la division d'élevage, les services vétérinaires étant privatisés. La fabrication et la maintenance du matériel sont désormais assurées par un réseau de forgerons. Les fonctions d'approvisionnement en matériel agricole et en bœufs de labour sont à la charge des paysans. Suite au fort endettement des organisations paysannes, les crédits matériels et bœufs ont été supprimés, ce qui rend l'accès à l'équipement difficile. Des retards dans l'exécution du calendrier agricole sont constatés dus à la mauvaise condition alimentaire et sanitaire des bœufs en début de campagne. Pour y remédier, le projet Urdoc (Unité de recherche développement observatoire de changement) a mis au point et testé un ensemble de mesures permettant d'améliorer l'état des animaux de trait. Leur diffusion se fait dans le cadre de l'approche " conseil à l'exploitation familiale ». La pérennisation de l'impact positif de la traction animale dépendra de l'engagement des différents acteurs au renforcement des services d'appui : conseil, crédit, entretien/fabrication de matériel et soins vétérinaires.
\end{abstract}

\section{INTRODUCTION}

Parmi les grands périmètres irrigués de l'Afrique de l'Ouest, l'Office du Niger $(\mathrm{ON})$ constitue un cas d'exemple où la traction animale joue un rôle clé dans la mise en valeur des terres, en étant l'un des principaux facteurs pouvant expliquer la rentabilité des exploitations familiales. Actuellement, la grande majorité des paysans ont recours à la traction bovine pour la préparation du sol et à la traction asine pour le transport. A part quelques tracteurs et plusieurs centaines de motoculteurs, la motorisation est peu utilisée pour la

\footnotetext{
1. Urdoc, BP 11, Niono, Mali

E-mail : hkassambara@yahoo.fr

2. Cirad, département Tera, Ambassade de France, BP 898, N’Djaména, Tchad

E-mail : paul.kleene@cirad.fr
}

culture. Par contre, les travaux postrécolte de battage et de décorticage de riz sont entièrement motorisés, faisant appel à de petites unités de transformation, exploitées par des organisations paysannes (OP) et des privés, le plus souvent producteurs eux-mêmes. En conséquence, la zone compte un grand nombre d'animaux de trait et d'équipements de traction animale. L'efficacité de leur utilisation dépend de l'attention que les producteurs, les artisans, les conseillers et les services d'appui et de recherche veulent et peuvent porter à ce capital.

Le présent article retrace l'historique de la traction animale à l'ON et fait un état des lieux de sa situation actuelle, sa force, ses contraintes et les nouveaux défis posés. Il est complété par la communication de Djire (3) sur la fabrication et l'entretien du matériel par les forgerons. La présentation de la zone ci-après sert à l'ensemble des deux documents. 


\section{PRESENTATION DE LA ZONE OFFICE DU NIGER}

L'ON, créé en 1932, est situé dans la partie occidentale du delta central du fleuve Niger, appelé delta mort. Le potentiel des terres irrigables par gravitation serait d'environ 1105000 ha (14). En 2001-02, 66030 ha ont été exploités (tableau I), dont 82,4 p. 100 en riz d'hivernage en casiers, 7,8 en riz d'hivernage hors casiers et 9,8 p. 100 en riz de contre-saison. Ces terres sont irriguées à partir du barrage de Markala et réparties entre les zones de production de Niono, N'Débougou, Molodo, Macina et Kouroumari (figure 1). En 2000, l'ON comptait 208 villages rassemblant 19470 familles attributaires pour une population totale estimée à 236116 personnes (1). Les principales cultures sont le riz en hivernage et les cultures maraîchères en contre-saison, dominées par l'échalote. Les superficies en riz de contre-saison sont limitées, face à l'alternative des cultures maraîchères (3 742 ha en 2001-02) et le manque de ressource en eau pendant la saison sèche. A quelques exceptions près, toutes les exploitations sont de type familial, en ayant la culture de riz hivernale comme activité commune de leurs membres. Le maraîchage de contre-saison est généralement le fait des femmes et des jeunes, travaillant pour leur propre compte. Les animaux de trait, bœufs pour le travail du sol et ânes pour le transport par charrettes, sont utilisés au niveau de l'exploitation. L'élevage, petits et gros ruminants, et volaille, joue un rôle important dans les systèmes de production, aussi bien au niveau commun qu'individuel. Hormis la tentative de grande mécanisation en régie dans les années 1950, le choix initial de la traction bovine comme moyen de cultiver les terres s'est maintenu jusqu'à nos jours (6). Cette option répond particulièrement bien aux caractéristiques physiques des terres de l'ON. Une pente générale, mais très faible dans le sens $\mathrm{SO}-\mathrm{NE}$, est interrompue par un microrelief souvent important au niveau des parcelles. Un (ré)aménagement du tertiaire en petits bassins $(0,10$ à 0,20 ha) entourés de diguettes est la condition sine qua non pour une irrigation et un drainage efficaces et économes en eau, à condition que le bassin soit bien plané. L'accès à de tels bassins est difficile pour des tracteurs, contrairement aux bœufs. Les bovins assurent la presque totalité des travaux de préparation du sol, tandis que les ânes occupent une place de choix dans le transport entre les villages et les champs, que ce soit pour les intrants, le matériel, les récoltes, le bois, le banco ou les personnes. Avant la restructuration intervenue en 1994, l'ON, en tant qu'entreprise étatique, gérait et exécutait en régie presque toutes les activités et services en amont et en aval de la production : de l'approvisionnement en intrants, jusqu'au battage, l'usinage et la commercialisation de riz. Il gérait l'eau et le foncier, assurait l'encadrement des «colons », contrôlait la gestion des coopératives (encadré 1). Actuellement, l'ON, établissement public à caractère industriel et commercial, a vu ses missions recentrées autour de : i) la gérance des terres et des infrastructures ; ii) la gestion de l'eau ; et iii) le conseil rural.

Ce changement du contexte institutionnel a fortement modifié la politique de l'entreprise par rapport à la traction animale, dans la mesure où elle n'intervient plus directement, ni dans la fabrication de matériel, ni dans l'approvisionnement en bœufs et en équipements, ni dans les services vétérinaires pour les soins des animaux.

\section{- HISTORIQUE DE LA TRACTION ANIMALE A L'OFFICE DU NIGER}

\section{De la création de l'ON à 1948 : introduction de la traction bovine (source: Schreyger, 1984, Steiner)}

L'introduction de la charrue et de la charrette bovine a été faite dès 1932 dans la zone de Macina et à partir de 1934 dans la zone de Niono. Elle se substitua ainsi à la daba, seul instrument utilisé pour la préparation du sol. Cette option permettait d'effectuer le labour plus rapidement et de réduire la pénibilité du travail du sol. L'introduction se faisait au travers des associations agricoles indigènes (AAI), créées dans chaque centre de colonisation (village). L'adhésion aux AAI était obligatoire, les méthodes utilisées étaient coercitives. Ainsi, l'ON remettait, sous condition de remboursement, à chaque famille quatre bœufs de labour et une charrue. En 1937, on comptait 2,7 bœufs de labour par charrue pour une superficie totale aménagée de 6017 ha (tableau I). L'insuffisance de l'assistance vétérinaire face aux épidémies et le faible niveau technique des colons dans la conduite des animaux de trait ont fortement limité l'utilisation des bœufs de labour à l'époque. Ils étaient incapables de respecter à temps l'exécution des travaux du sol. Ce problème, conjugué à d'autres, comme la faible maîtrise de la fertilisation, le coût élevé des intrants et la démotivation des colons du fait des mauvaises conditions de vie à l'ON, a été à l'origine des résultats économiques médiocres. Dans les stations d'essai, les rendements s'élevaient en moyenne à $1572 \mathrm{~kg} / \mathrm{ha}$ pour le coton et à $2400 \mathrm{~kg} /$ ha pour le riz. Mais les paysans ne récoltaient en moyenne que $612 \mathrm{~kg}$ de coton et $1490 \mathrm{~kg}$ de riz par hectare. L'analyse faite de cette situation a été à l'origine de l'adoption de la politique de

\section{Encadré 1}

\section{Quelques GRANDES DATES DE L'OfFICE du NiGer *}

1932 : création de l'Office du Niger (ON), début des aménagements dans la zone de Macina et de Niono

1947 : mise en service du barrage de Markala permettant de parfaire l'aménagement des zones de Macina et de Niono et les extensions aux zones de N'Débougou, de Molodo et de Kouroumari

1949 : création du Centre rizicole mécanisé de Molodo 1970 : abandon de la culture de coton

1978 : première table ronde avec des bailleurs de fonds pour la réhabilitation de l'ON

1978-1981 : attribution des terres des périmètres irrigués sur la base de 4 ha par charrue

1982 : début des réhabilitations dans les secteurs de Niono par le projet Arpon

1984 : création des premières associations villageoises, fournissant des services de battage de riz, de crédit campagne (semences et engrais) et de crédit moyen terme pour le matériel agricole et les bœufs de labour (à travers I'ON ou des institutions financières)

1985 : suppression du crédit d'intrants agricoles accordé par l'ON, désormais fournis par le Fonds intrants agricoles (FIA), puis le Fonds de développement villageois (FDV) du projet Arpon ou par la Banque nationale pour le développement agricole (Bnda) (projet Retail)

1992 : le FDV est transformé en Fédération des caisses rurales mutualistes du Delta (Fcrmd), deux autres systèmes de financement décentralisés (SFD) s'installent

1994 : restructuration de l'ON dans le cadre du désengagement de l'Etat

1997 : début de nouveaux aménagements avec participation financière des attributaires

* D'après Bonneval et coll., 2002, Cirad 

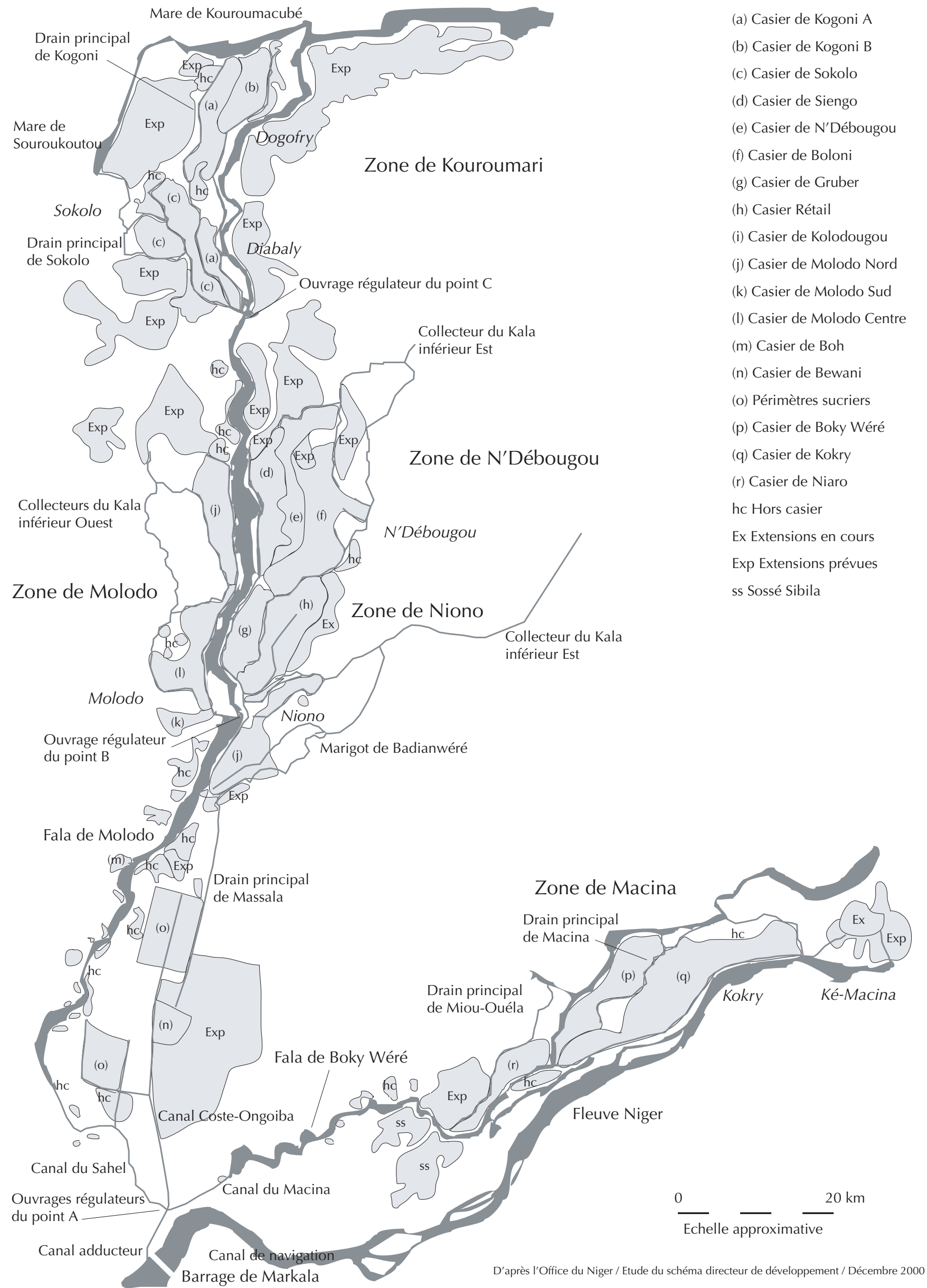

Figure 1 : carte des aménagements de la zone de l'Office du Niger. 


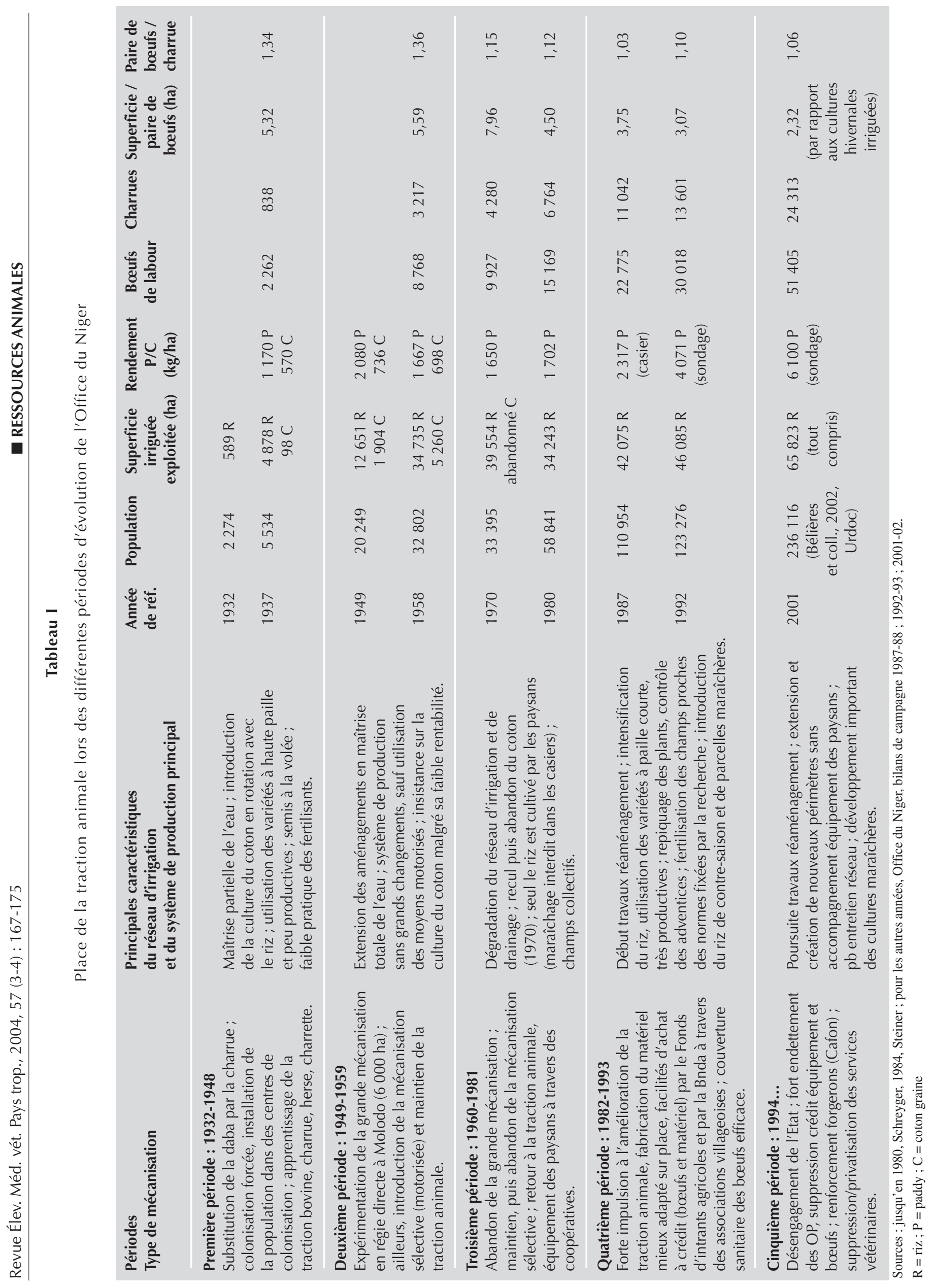


modernisation des techniques de culture basée sur l'emploi de la motorisation. Le but recherché était d'obtenir une augmentation de la production agricole dans un système intensif. Ce fut alors le début de la grande mécanisation.

\section{De 1949 à 1959 : introduction de la grande mécanisation (source : Schreyger, 1984, Steiner)}

Après l'achèvement du barrage de Markala en 1947, la grande mécanisation a fait son entrée dans la zone ON en 1949 par la création du centre rizicole mécanisé de Molodo. Dans cette zone de production de 6000 ha, toutes les opérations devaient être faites en régie, de façon motorisée. Le grand parc de machines comptait 150 tracteurs à chenilles et 450 autres appareils, tandis que l'effectif du personnel salarié était de 700 employés. En dépit de l'amélioration des techniques culturales, on s'est rendu compte que l'accroissement des rendements ne suffisait même pas pour couvrir les frais d'exploitation (salaires, prix du carburant et entretien des équipements). C'est ainsi qu'en 1957, le déficit se chiffra à environ 35 millions de francs. Ainsi, selon les concepteurs, en dix ans d'expérimentation, la grande mécanisation a été un échec total dans la zone de Molodo.

En même temps dans les autres secteurs, la forme de production a été celle de la mécanisation sélective en complément à la traction bovine. La motorisation, gérée en régie, intervenait en cas de besoin pour effectuer du labour profond, facturé au colon. Le coût de ces travaux pesait très lourdement sur les comptes d'exploitation, atteignant jusqu'au tiers de la valeur du produit brut en paddy. Quant à la traction bovine, le niveau d'équipement des colons lors de la campagne 1958-59, donnait le même ratio que celui de 1937, c'està-dire 2,7 bœufs de labour par charrue en moyenne (tableau I).

\section{De 1960 à 1984 : retour à la traction animale (source : Schreyger, 1984, Steiner)}

L'échec de la grande mécanisation a impliqué un retour à la traction animale. Dans la période qui suivit l'indépendance en 1960, l'ON a donné une nouvelle impulsion aux paysans pour s'équiper en matériel et en bœufs au travers du crédit moyen terme payé par annuité, en passant par des coopératives qui avaient remplacé les AAI. Leur adhésion était également obligatoire et leur fonctionnement n'était qu'un léger mieux par rapport au passé. La production restait fortement encadrée, avec livraison obligatoire de la production du paddy à l'ON, qui gardait le monopole du battage, de l'usinage et de la commercialisation. Le paysan ne recevait que le solde du compte, souvent minime après soustraction des prélèvements, la redevance eau, les crédits intrants, et l'annuité en cas de crédit équipement. Le début de cette période fut marqué par l'introduction de champs collectifs, avec participation obligatoire, abandonnés après la fin de la période socialiste, en 1968. Jusqu'en 1970, le retour à la traction animale et l'extension des superficies et du nombre de colons n'ont pas été suivis par un équipement suffisant des paysans. Au bilan de la campagne 1970-71 (tableau I), le ratio superficie par paire de bœufs était de 7,96 ha, ce qui dépassait de loin la norme de 3 ha.

Cette situation s'est redressée lors de la décennie suivante, lorsque le meilleur climat économique aidant, le ratio superficie par paire de bœufs s'est nettement amélioré et a atteint 4,50 ha en 1980-81. La sécheresse des années 1973 et 1974 et les épidémies ont occasionné des mortalités et des ventes élevées de bovins, réduisant leur effectif. Grâce à l'irrigation, la zone a relativement bien survécu à cette période et les pertes en animaux de trait ont pu être plus que compensées. L'ON s'occupait lui-même de l'achat des bœufs et de leur dressage dans des centres spécialement créés à cet effet. Le matériel agricole, charrues TM (tropicale), herses et charrettes asines étaient assemblées dans l'unique usine de matériel agricole, la Société malienne d'étude et de construction de matériel agricole (Smecma) à Bamako. Cependant, la même période a été caractérisée par une dégradation progressive du réseau d'irrigation et de drainage, par manque d'entretien. Les rendements moyens en paddy obtenus par les paysans oscillaient entre $1650 \mathrm{~kg} / \mathrm{ha}$ en 1970-71, $1702 \mathrm{~kg} / \mathrm{ha}$ en 1980-81 et $1680 \mathrm{~kg} / \mathrm{ha}$ en 1984-85 (14). Comparés aux rendements des années 1940 et 1950, aucun progrès notable n'avait été fait. Outre les insuffisances et la dégradation du réseau, la faiblesse des rendements s'explique par le système de production extensif mis en place, caractérisé par :

- la pratique du semis à la volée ;

- une faible maîtrise des adventices et du riz sauvage ;

- une fertilisation des champs très insuffisante ;

- l'utilisation de variétés rustiques mais peu productives.

Pour le coton, les problèmes de parasitisme et du drainage ont finalement entraîné l'abandon de cette culture en 1970. Le riz est resté comme seule spéculation, le maraîchage étant interdit dans les casiers. Le manque de rentabilité des exploitations paysannes a été aggravé par un manque de rentabilité au niveau de l'ON qui a cumulé des déficits d'exploitation d'année en année, comblés par le budget de l'Etat. Devant cette situation, le gouvernement malien a lancé un appel international en 1978 pour la relance de la production rizicole dans la zone ON. La réponse s'est fait attendre et s'est limitée, dans un premier temps, à des initiatives de la coopération néerlandaise, dont la plus importante a été le démarrage du projet Amélioration de la riziculture paysanne en zone ON (Arpon) en 1982. Elle a été suivie par d'autres projets et financements, dont le projet Retail (coopération française), lancé en 1986.

\section{De 1982 à 1994 : nouvel essor de la traction animale}

A partir de 1982, l'ON est entré dans une phase de réhabilitation du réseau et d'intensification de la riziculture, lors de laquelle une attention particulière a été portée à la traction animale, notamment à travers le projet Arpon. De nouveaux matériels ont été introduits, fabriqués à partir de la matière première et de semi-fabrications importées des Pays-Bas par l'Atelier d'assemblage de matériel agricole (Aama). Il s'agit notamment de la charrue et de la herse Rumptstadt, appelées «petère » par les paysans (d'après le premier chef de projet Arpon, Peter Bolt), des charrettes, puis des barres et des pelles de planage tirées par les bœufs, appelées «bœufscrapers » et « bœufdozers ». La responsabilisation paysanne est intervenue en 1984 par la création des premières associations villageoises (AV).

En 1985 s'est opérée la suppression du crédit intrants agricoles accordé par l'ON et la création du Fonds intrants agricoles (FIA) par le projet Arpon. L'objectif était le renforcement de la capacité des organisations paysannes nouvellement créées, tout en favorisant l'augmentation de la production et de la productivité du riz (14). Outre la fourniture de crédits pour l'approvisionnement des riziculteurs en intrants, le FIA intervenait avec du crédit moyen terme pour la fourniture de matériel agricole et de bœufs de labour de façon très dynamique. Des dizaines de milliers d'unités de matériel ont été injectées dans la zone, dont une bonne partie est encore opérationnelle. Le FIA * approvisionnait les associations villageoises, et plus tard aussi des privés, en petites batteuses motorisées, de marque Votex, qui ont entièrement repris la fonction de battage assurée jusque-là par l'ON. L'introduction de petites décortiqueuses motorisées, d'abord de marque Votex, plus tard de marques chinoises, complétait ce triptyque de la mécanisation décentralisée : traction animale pour le travail du sol et le transport, petite motorisation pour le battage et le décorticage. Ainsi, dans une situation de marché de travail abondant et un manque de

* Il s'est transformé plus tard en Fonds de développement villageois (FDV), à la base de la Fédération des caisses rurales mutualistes du Delta (Fcrmd), créée en 1994. 
devises au niveau macroéconomique, le riz se produisait au moindre coût et un maximum de valeur ajoutée était récupéré au niveau des paysans.

En 1992, la situation de l'équipement agricole dans la zone affichait un ratio de 3,07 ha par paire de bœufs de labour (tableau I), proche de la norme de 3 ha, considérée comme un bon indicateur pour le respect du calendrier agricole. De plus, l'assistance vétérinaire et zootechnique donnée par l'ON et appuyée par le projet Arpon permettait aux paysans de mieux entretenir leurs bœufs, plus aptes à exécuter le travail du sol.

L'interférence des autres facteurs de l'intensification ont largement contribué à l'augmentation des rendements de riz paddy qui sont passés de 2 à 5 t/ha en moyenne. Ces autres facteurs ont été :

- les réaménagements et le découpage des parcelles en bassins plus petits ;

- la diminution de la taille des superficies par famille attributaire ; - l'utilisation des variétés améliorées à courte paille ;

- la pratique du repiquage ;

- l'utilisation des fertilisants à forte dose.

C'est dans ce contexte qu'est intervenue la restructuration de l'ON en 1994.

\section{CONTEXTE ACTUEL DE LA TRACTION ANIMALE}

Les importants efforts déployés pendant la période 1982-94 avaient permis aux exploitations familiales d'atteindre un seuil de rentabilité appréciable. Après la dévaluation du franc CFA début 1994, le riz produit à l'ON était devenu compétitif avec le riz asiatique importé. Tel n'était pas le cas de l'entreprise ON, structure devenue trop lourde, chroniquement déficitaire. Beaucoup de tâches avaient déjà été partiellement reprises par le secteur associatif (les $\mathrm{OP})$, bancaire et privé ; d'autres allaient suivre après la restructuration. Ce désengagement de l'Etat s'est résumé par une limitation des mandats de l'ON (voir plus haut). En ce qui concerne la traction animale, l'ON s'est retiré de tout service d'appui. L'entretien du matériel agricole a été entièrement transféré aux forgerons et à leur coopérative, la Coopérative artisanale des forgerons de l'ON (Cafon) (3). L'atelier d'assemblage (Aama) a été repris par la Smecma privatisée, mais ses activités de fabrication de matériel de culture attelée ont été reprises par les forgerons de la Cafon.

En matière de soins vétérinaires, la suppression du service santé animale a créé un vide qui n'a été rempli que très partiellement par l'unique mandataire de la zone, dont les services sont restés insuffisants en volume et en rapport qualité/prix. A partir de 1999, l'association Yiriwa-So a introduit des soins contractualisés qui répondent de façon adéquate aux besoins des agroéleveurs. Toutefois, le rayon d'action est encore trop faible et la zone continue à souffrir d'un manque de couverture sanitaire.

Quant au financement des intrants et des équipements, ce service est désormais assuré, en principe, par les «caisses », institutions financières décentralisées, installées dans la zone (Fcrmd, Caisses associatives rurales d'épargne et de crédit [Carec] et réseau Nyésigiso). La Banque nationale pour le développement agricole (Bnda) a également une agence à Niono, mais elle joue le rôle d'institution de refinancement vis-à-vis des caisses et intervient rarement pour des crédits accordés directement à des producteurs (19).

La restructuration de l'ON a coïncidé avec la libéralisation du commerce du riz qui, mal préparée, a ouvert la porte à des malversations à grande échelle. Beaucoup d'associations villageoises, dont certaines étaient déjà très endettées, ont subi des pertes si importantes que leur survie a été menacée. Ainsi, à partir de 1995 le crédit équipement moyen terme, basé sur la caution solidaire des AV, a été supprimé par l'ensemble des institutions financières. Malgré les bons résultats obtenus par les centres de prestations de services* en échelonnement, puis remboursement des dettes, cette situation reste toujours bloquée. En conséquence, depuis huit ans les paysans dépendent de l'autofinancement pour l'achat de leur matériel agricole et des animaux de trait. Les exploitations bien établies remplacent régulièrement leurs bœufs (4 289 en 2001-02) et ânes, mais on constate un vieillissement de leur matériel. Ce sont surtout les nouvelles exploitations, celles issues d'éclatement de familles ou installées dans de nouveaux aménagements qui n'arrivent pas à s'équiper ou restent sous-équipées.

\section{Animaux de trait}

Les bœufs de labour sont soit achetés sur le marché, soit proviennent du troupeau de l'exploitant. Les bovins sont dominés par la race Peule et la race Maure. Le Peul est très sollicité par les exploitations agricoles du fait de son adaptabilité aux conditions climatiques de la zone. Le bœuf de race Peule est de grande taille, 1,30 à $1,50 \mathrm{~m}$ au garrot, longiligne et à l'âge adulte son poids varie de 280 à $350 \mathrm{~kg}$. Le poids moyen d'un bœuf de labour est de $285 \mathrm{~kg}$ (20). Les ânes sont pour la plupart achetés auprès de vendeurs ambulants ; ils sont de petite taille, $0,80 \mathrm{~m}$ au garrot avec un poids variant de 80 à $100 \mathrm{~kg}$ à l'âge adulte.

En 2001-02 la zone comptait au total 51405 bœufs de labour et 18679 ânes (tableau I). D'après Bélières et coll. (1), les exploitations attributaires disposent en moyenne de 2,5 bœufs de trait et d'un âne. Une partie de ces exploitations (26 p. 100) disposent de deux paires de bœufs ou plus et cumulent 60 p. 100 des animaux recensés. Toutefois, 31 p. 100 des exploitations attributaires ne possèdent pas de paire de bœufs et dépendent de prêts et de locations pour effectuer les travaux (coût total estimé à $60000 \mathrm{Fcfa} / \mathrm{ha}$ ). Il s'agit de petites exploitations, cultivant en moyenne 2,11 ha de superficie irriguée disponible (1). Il existe donc de fortes disparités entre exploitations de types différents.

D'une manière générale, les exploitations agricoles rencontrent de nombreuses difficultés dans la gestion des bœufs de labour. Leur alimentation en saison sèche est souvent déséquilibrée et insuffisante. Ils entament la campagne déjà trop maigres et finissent la période de travaux épuisés, n'ayant pas reçu de rations alimentaires ajustées.

En ce qui concerne les ânes, trois exploitations attributaires sur quatre ont au moins un âne. Souvent ils transportent des charges allant jusqu'à $700 \mathrm{~kg}$, ce qui dépasse de loin les normes conseillées (300 à $400 \mathrm{~kg}$ ). Il en est de même pour le temps d'exploitation. Ils travaillent en moyenne plus de $7 \mathrm{~h} /$ jour, alors que les normes ne prévoient que 3,5 à $4 \mathrm{~h} /$ jour. Leur harnachement est souvent inapproprié. Ce mode d'exploitation épuise l'âne rapidement et oblige le paysan à le remplacer vite, souvent en moins d'une année. Jusqu'à une date récente, les ânes ne faisaient l'objet d'aucun soin particulier. Or leur prix a connu une hausse importante ces derniers temps, passant de 40000 à 60000 Fcfa ou plus, ce qui peut expliquer le gain d'intérêt récent pour un meilleur entretien des ânes de la part de leurs propriétaires.

\section{Matériel de traction animale}

La gamme de matériel est celle mise en place par le projet Arpon à partir de 1984 (voir plus haut), fabriquée par les forgerons de la Cafon en fonction de la demande (3). L'équipement de base,

* Les centres, au nombre de cinq, ont été créés à partir de 1995 et sont gérés par les OP adhérentes; en 2002, ils ont créé la Fédération des centres de prestation de services en zone $\mathrm{ON}$ 
constitué par la charrue, la herse et la charrette asine, donne satisfaction aux paysans. En 2001-02, le ratio nombre de paires de bœufs par charrue était de 1,06 et celui de la superficie par paire de bœufs de labour était de 2,32 ha (tableau I).

L'utilisation de la charrue non réversible et la pratique du labour à la Fellemberg sont à l'origine du problème récurrent de planage des bassins. En alternant avec un labour en planches ou un labour à l'inverse, certains paysans arrivent à s'en sortir (7). Pour y remédier le projet Retail, puis l'Urdoc ont cherché des remèdes, notamment par des tests de charrue réversible tourne-oreille, de charrue à claire-voie et de herse rotative $(9,10,11)$. Depuis 2000 , des expérimentations ont été reprises par l'Institut d'économie rurale (IER) et le Centre de coopération internationale en recherche agronomique pour le développement (Cirad) et ont abouti à des résultats probants. La vulgarisation de la herse roulante intervient en 2004. En collaboration avec la Cafon, un nouveau modèle de la barre planeuse a été développé et testé par l'Urdoc. En remplaçant la planche pleine par une lame en fer dentée, on évite le bourrage et on obtient une meilleure répartition de la boue. Ce nouveau modèle connaît un certain succès, grâce à des démonstrations dans le cadre de l'approche conseil à l'exploitation familiale (CEF) (voir plus loin) et un début de diffusion à travers les centres de prestation de services. Les deux partenaires ont également travaillé ensemble pour tester et prévulgariser une botteleuse pour la paille de riz.

\section{Matériels motorisés de traction}

L'introduction de motoculteurs depuis 1985 connaît un certain succès, leur efficacité est surtout reconnue pour la mise en boue (puddlage) et le transport. En 2000, 1,5 p. 100 des attributaires avaient du matériel de traction motorisé. Il s'agissait des plus grandes exploitations disposant de 8,3 p. 100 de bovins et de 4 p. 100 des attelages. Elles disposaient de trois attelages en moyenne pour une superficie irriguée totale de 12,33 ha, soit un attelage pour environ 4 ha (1). Cela montre que dans ces exploitations le matériel motorisé ne remplaçait pas la traction animale, mais y était complémentaire.

\section{ACTIONS MENEES EN APPUI A LA CULTURE ATTELEE}

\section{Amélioration de la performance des bœufs}

La pratique paysanne dans la gestion des bœufs ayant été jugée insuffisante a conduit le projet Urdoc à entreprendre des actions de recherche participative pour leur amélioration. Elles ont porté sur l'alimentation, les soins de santé et la conduite et ont été réalisées en quatre étapes :

- l'étude diagnostic sur la complémentation alimentaire (4);

- l'étude diagnostic du suivi sanitaire (8);

- l'analyse des pratiques de conduite ;

- la proposition de normes et de test sur le terrain.

Les résultats obtenus sont décrits ci-après.

\section{Sur le plan alimentaire}

Le but a été de proposer des rations répondant aux besoins et à faible coût, et s'appuyant sur les pratiques paysannes basées sur les sous-produits de la riziculture. Pendant la période d'entretien, à partir des besoins, estimés à 2,6 UF et $240 \mathrm{~g}$ de matière azotée digestible (MAD) par jour pour un bœuf de $300 \mathrm{~kg}$ (18), il ressort que la paille de riz ( $5 \mathrm{~kg} / \mathrm{jour}$ ) et le son de riz (4 kg/jour) suffisent en complément au pâturage naturel, dont il est difficile de quantifier l'apport. Pendant la période des travaux, à partir des besoins estimés à 4,8 UF et $396 \mathrm{~g}$ MAD par jour pour un bœuf de $300 \mathrm{~kg}$
(11), l'adjonction d'un kilogramme de mélasse à la ration d'entretien suffit, étant donné que le pâturage naturel est plus riche pendant cette période. La difficulté de cette ration est la disponibilité de la mélasse. Les deux sucreries de la zone la produisent, mais la tendance est de transformer le maximum en alcool, parce qu'il est plus rentable. Le circuit de distribution de la mélasse, qui existait du temps du projet Arpon, n'est plus fonctionnel. Des minéraux (pierres à lécher) doivent être fournis à volonté, tant pour l'entretien que pour le travail.

A noter que dans les deux cas ces rations sont différentes de celles proposées par l'IER, basées sur des recherches menées en station et qui incluent l'urée et l'ABH, aliment bétail à base de tourteau et coque de coton (5). Elles posent des problèmes à cause de leur coût élevé et de la disponibilité des intrants sur le marché.

\section{Sur le plan sanitaire}

Le plan de prophylaxie sanitaire, élaboré en 1999 et testé auprès des exploitants, a donné des résultats probants (tableau II). Il s'adresse aux principales maladies bovines rencontrées dans la zone. Les périodes propices pour les traitements sont fonction du calendrier agricole et pastoral. Il est appliqué dans le cadre de la contractualisation des soins vétérinaires.

\section{Tableau II}

Plan de prophylaxie sanitaire pour les bovins de la zone Office du Niger

\begin{tabular}{|c|c|c|}
\hline $\begin{array}{l}\text { Principales } \\
\text { maladies }\end{array}$ & $\begin{array}{l}\text { Nombre de } \\
\text { traitements / an }\end{array}$ & $\begin{array}{l}\text { Période des } \\
\text { traitements (mois) }\end{array}$ \\
\hline Distomatose & 2 fois & Décembre - juillet \\
\hline Trypanosomose & $\begin{array}{l}\text { Au besoin } \\
\text { avec un curatif }\end{array}$ & \\
\hline Gale & $\begin{array}{l}\text { Au besoin } \\
\text { avec un curatif }\end{array}$ & $\begin{array}{l}\text { Désinfecter } \\
\text { les locaux }\end{array}$ \\
\hline $\begin{array}{l}\text { Parasitoses } \\
\text { internes }\end{array}$ & 2 fois & $\begin{array}{l}\text { Début et fin } \\
\text { hivernage }\end{array}$ \\
\hline Péripneumonie & 1 fois & $\begin{array}{l}\text { Novembre } \\
\text { - février }\end{array}$ \\
\hline Pasteurellose & 2 fois & Février - juillet \\
\hline $\begin{array}{l}\text { Charbon } \\
\text { symptomatique }\end{array}$ & 2 fois & $\begin{array}{l}\text { Entre février } \\
\text { et mars }\end{array}$ \\
\hline
\end{tabular}

Source : Urdoc, 2003, Guide

\section{Sur le plan organisation du travail}

Les recommandations suivantes ont été élaborées : en début du travail du sol, utiliser les bœufs sur de petites superficies, durant au maximum quatre heures; le travail doit commencer tôt le matin, entre 5 et $6 \mathrm{~h}$, et prendre fin vers 9 à $10 \mathrm{~h}$, pour éviter les heures chaudes de la journée; au fur et à mesure que la campagne agricole avance, augmenter progressivement la superficie et le temps de travail journalier.

\section{Diffusion à travers le conseil à l'exploitation}

Un module sur la gestion des bœufs de labour a été produit sur la base de ces références, des données bibliographiques et autres connaissances. Il fait partie du Guide du conseiller aux exploitations familiales élaboré par le projet Urdoc et ses partenaires (18). 
Il est composé de sept thèmes (tableau III) et sert comme support aux conseillers en gestion dans l'animation de séances « conseil à l'exploitation» avec des groupes de paysans volontaires. Il est le produit d'un test fait au préalable auprès de différents groupes d'agroéleveurs. Les mots techniques sont homologués à la langue vernaculaire locale. Les producteurs participent ainsi à la validation des outils.

\section{Tableau III}

Thèmes du module entretien des bœufs de labour

$\begin{array}{ll}\text { Module } & \text { Thèmes } \\ \begin{array}{ll}\text { Entretien } \\ \text { des bœufs } \\ \text { de labour }\end{array} & \text { Détermination de l'âge des bovins } \\ & \text { Critères de choix d'un bon bœuf de labour } \\ & \text { Habitation des bœufs de labour } \\ & \begin{array}{l}\text { Alimentation complémentaire des bœufs } \\ \text { de labour }\end{array} \\ & \text { Soins sanitaires des bœufs de labour } \\ & \begin{array}{l}\text { Estimation et gestion des ressources } \\ \text { fourragères de l'exploitation agricole }\end{array} \\ & \text { Technique d'embouche des bœufs de reforme }\end{array}$

Source : Urdoc, 2003, Rapport

Les conseillers intéressés par le module suivent d'abord une formation en salle sur l'animation de groupes sur ces thèmes. Ensuite, ils vont sur le terrain, accompagnés d'un agent du projet Urdoc pendant leur période de formation. Les groupes CEF sont constitués de paysans volontaires, au nombre de 7 à 15 , alphabétisés en langue nationale, qui se rencontrent régulièrement à des jours fixes. Les séances, d'une durée de 2 à $3 \mathrm{~h}$, commencent par une brève introduction sur le thème du jour. Ensuite les participants expliquent leurs pratiques actuelles. Les forces et les faiblesses de ces pratiques sont analysées et discutées dans le groupe. Puis, les références techniques de la recherche sont exposées, discutées et adaptées, si nécessaire, par exemple si les paysans disposent d'autres ressources fourragères comme les fanes de niébé ou d'arachide. Elles serviront de base de calcul pour la prévision des réserves de sous-produits agricoles et des forages à constituer après la récolte, pour subvenir aux besoins des animaux de l'exploitation jusqu'à la fin de la période des travaux. Ce module est utilisé par les conseillers CEF des centres de prestation de services, les conseillers ruraux de l'ON et les agents de l'association Yiriwa-So, auprès de leurs différents groupes de clients. Actuellement, 21 groupes, soit environ 200 personnes pour la plupart des chefs d'exploitation ou leurs adjoints, ont participé à ce module (17).

\section{Renforcement de la capacité des forgerons}

La présence d'un réseau performant de forgerons, réunis dans la Cafon, constitue l'un des piliers de l'appui à la traction animale dans la zone décrite par Djire (3).

\section{- CONCLUSION ET PERSPECTIVES}

L'avènement du processus d'intensification à partir des années 1980, accompagné d'appuis importants et efficaces à la traction animale, a permis une augmentation forte des rendements obtenus par les paysans et a rendu la riziculture rentable au niveau des exploitations. La suppression du crédit équipement et du volet élevage de l'ON a bouleversé le contexte de la traction animale. Sur fonds propres, les paysans se trouvent actuellement face au secteur privé et associatif pour l'achat et l'entretien de leurs équipements, les soins sanitaires des animaux de trait et les conseils zootechniques (hormis les conseils fournis par les agents du service conseil rural de l'ON, qui est un service public). Les paysans sont de plus en plus demandeurs de conseils, mais ils répugnent à en payer le prix, même s'il est subventionné. L'absence de financement pour l'équipement est un grand handicap pour les nombreux paysans qui rejoignent la zone, en pleine expansion avec en moyenne 3000 ha/an de nouveaux aménagements depuis 1998. Le parc de matériel existant est vieillissant, alors que la capacité locale pour le renouveler existe. Dans tous les cas, le problème de sous-équipement et d'entretien des animaux de trait est une réalité pour les petites exploitations. Des retards dans l'exécution du calendrier agricole sont souvent constatés. Le problème du mauvais planage des parcelles ne peut pas être résolu sans innovation des techniques culturales et du matériel utilisé. La surconsommation d'eau qui en résulte contribue au mauvais drainage, qui est l'un des principaux problèmes du réseau hydraulique et qui conduira à terme à une dégradation des sols.

Le moment semble être bien indiqué pour des innovations en matière d'appui à la traction animale. Les actions pourraient s'articuler autour de cinq axes :

- la réintroduction d'un crédit équipement, basé sur des principes de financement sain, incluant l'appui conseil aux bénéficiaires, pour le renouvellement du parc existant et surtout l'équipement des nouveaux venus dans les périmètres nouvellement aménagés ;

- le renforcement de la recherche-développement sur des techniques de planage des parcelles et la mise au point des équipements appropriés ;

- l'appui durable aux forgerons dans la confection locale des nouveaux équipements, y compris des harnachements améliorés, et dans leur diffusion à travers les $\mathrm{OP}$;

- le renforcement des services vétérinaires contractualisés et d'appui conseil à la traction animale par des subventions ciblées, au travers des organisations paysannes et professionnelles, respectant des cahiers de charge précis ;

- la création d'un cadre de coordination entre les différents acteurs de la filière traction animale.

Dans ce cas, l'ensemble des acteurs (ON, organisations des paysans et des forgerons, institut de recherche, projets d'appui, institutions financières) devraient s'investir en faveur de la réalisation de ces objectifs ; il y va de l'avenir de la traction animale actuellement incontournable dans le système de production pratiqué par les exploitations familiales.

\section{BIBLIOGRAPHIE}

1. BELIERES J.F., COULIBALY Y.M., KEITA A., SANOGO, M.K., 2002. Caractérisation des exploitations agricoles de l'Office du Niger, résultats d'une enquête des exploitations dans un échantillon de villages. Niono, Mali, Urdoc, 100 p. + annexes.

2. BONNEVAL P., KUPER M., TONNEAU J.-P., 2002. L'Office du Niger, grenier à riz du Mali, le fil de l'eau et la part des hommes. Montpellier, France, Cirad, $250 p$

3. DJIRE O., 2004. Le service de fabrication et de maintenance d'équipements agricoles par les forgerons au Mali. In: Actes atelier Traction animale et stratégies d'acteurs: quelle recherche, quels services face au désengagement de l'Etat? Bobo-Dioulasso, Burkina Faso, 17-21 nov. 2003. Revue Elev. Méd. vét. Pays trop., 57 (3-4). 
4. DOUMBIA A.A., 1997. Supplémentation alimentaire des bœufs de labour, test de deux suppléments dans la zone Office du Niger, Niono. Mémoire Ingénieur, IPR, Katibougou, Mali, 42 p. + annexes.

5. IER, 2002. Rapport annuel 2002. Niono, Mali, Crra.

6. JAMIN J.-Y., 1994. De la norme à la diversité : I'intensification rizicole face à la diversité paysanne dans les périmètres irrigués de l'Office du Niger. Thèse Doct. INA-PG / Montpellier, Cirad-sar, France, 255 p. + annexes.

7. JAMIN J.-Y., KEITA M., SOW M., 1990. Mécanisation en culture attelée dans l'intensification de la riziculture irriguée. Le projet Retail de l'Office du Niger. Cah. Rech. Dév., nº 28.

8. KASSAMBARA H., OMBOTIMBE S.S., 1998. Etude diagnostic du suivi sanitaire des bœufs de labour en zone Office du Niger. Le cas du Kala inférieur. Mémoire Ingénieur, IPR, Katibougou, Mali, 55 p. + annexes.

9. LE LOUS R., 1986. Compte rendu de mission machinisme agricole. Niono, Mali, Office du Niger, projet Retail, 46 p.

10. LE LOUS R., 1987. Essais de petits matériels de riziculture sur le projet Retail. Niono, Mali, Office du Niger, projet Retail, 17 p.

11. LE THIEC G., 1996. Agriculture africaine et traction animale. Montpellier, France, Cirad-sar, 355 p.
12. OFFICE DU NIGER, 1988. Bilan de campagne agricole 1987/1988. Ségou, Mali, Office du Niger, 22 p. + annexes.

13. OFFICE DU NIGER, 1993. Bilan de campagne agricole 1992/1993. Ségou, Mali, Office du Niger, 9 p. + annexes.

14. OFFICE DU NIGER, 1997. La restructuration de I'Office du Niger, contribution de Arpon III, coopération néerlandaise. Ségou, Mali, Office du Niger, 153 p.

15. OFFICE DU NIGER, 2002. Rapport bilan, saison et contre saison 2001/2002. Ségou, Mali, Office du Niger, 39 p. + annexes

16. SCHREYGER E., 1984. L'Office du Niger au Mali ; la problématique d'une grande entreprise agricole dans la zone du Sahel. Wiesbaden, Allemagne, Steiner, 394 p.

17. URDOC, 2003. Rapport d'activités 2002. Niono, Mali, Urdoc, 67 p.

18. URDOC, 2003. Guide du conseiller aux exploitations familiales. Productions animales. Niono, Mali, Urdoc, 43 p.

19. WAMPFLER B., 2003. Coordination et pérennisation des services autour du financement de l'agriculture familiale dans la zone Office du Niger (Mali). Montpellier, France, Cirad, 66 p. + annexes. (Tera $\mathrm{n}^{\circ} 27 / 03$ )

20. WILSON R.T., 1988. La production animale au Mali-Central : études à long terme sur les bovins et les petits ruminants dans le système agropastoral. Rapport de recherche. Addis Abeba, Ethiopie, Cipea, 118 p.

\section{Summary}

Kassambara H., Kleene P. Draft Animal Power at the Office of Niger in Mali. From State Management to State Disengagement

In the early 1930s, the Office of Niger started its farming operations with the use of cattle power. In 1949 began large scale mechanization, which ended in failure ten years later. Since then, draft animal power has become again the major energy source for soil labor and transportation. Until the early 1980s, the production systems were based on extensive techniques that led to average paddy yields that rarely reached beyond two tons per hectare. Starting in 1982, a new dynamic emerged from Office of Niger activities. Draft animal power was developed thanks to an easier access to credit, betteradapted made-on-site equipment, and adequate veterinary services. Average paddy yields reached four to six tons per hectare, depending on the service provided. After the 1994 Office of Niger restructuration, the animal husbandry division was cut out as a result of veterinary services privatization. Manufacturing of implements as well as maintenance are now performed by a blacksmiths' network. Peasants are financially responsible for supplying farm equipment and draft animals. Following the heavy debt of farmers' associations, credit facilities for implements and oxen were stopped, increasing thus the difficulty to get access to animal traction. Bad feed and health conditions of cattle at the beginning of the crop year result in delays in the application of the farmwork calendar. To remedy this situation, the URDOC project developed and tested a series of measures aimed at improving the welfare of draft animals. The results are available in the form of technical notes and teaching modules, which are circulated within the framework of "support/advice at the family farm level". The success of these measures will depend on the engagement of the various actors to provide support/advice and veterinary services, as well as to revive manufacturing, maintenance and credit for animal traction implements.

Keywords: Animal power - Agricultural policies - Delta Niger river - Mali.

\section{Resumen}

Kassambara H., Kleene P. La tracción animal en la Oficina del Níger en Malí: de la colonia al desentendimiento del Estado

La Oficina del Níger comenzó su actividad a principios de los años 1930 con la tracción bovina. La gran mecanización, introducida a partir de 1949, acabó diez años más tarde en un fracaso. Desde entonces, la tracción animal volvió a ser la principal fuente de energía para el trabajo del suelo y el transporte. Hasta inicios de los años 1980, los sistemas de producción estaban basados sobre técnicas extensivas, conduciendo a rendimientos medios de arroz paddy que sobrepasaban raramente 2 t/ha. A partir de 1982, se instauró una nueva dinámica en las actividades de la Oficina del Níger. La tracción animal se desarrolló enormemente, gracias a nuevas facilidades de crédito, al material mejor adaptado, fabricado in situ y a los servicios veterinarios adecuados. Los rendimientos medios de paddy pasaron de 4 a 6 t/ha, según el tipo de instalación. La reestructuración de la Oficina del Níger, llevada a cabo en 1994, trajo consigo la supresión de la división de cría, los servicios veterinarios se privatizaron. La fabricación y el mantenimiento del material son desde entonces asegurados por una red de herreros. Las funciones de abastecimiento en material agrícola y en bueyes de tiro están a cargo de los campesinos. En vista del gran endeudamiento de las organizaciones campesinas, los créditos materiales y los bueyes fueron eliminados, dificultando el acceso a los equipos. Se constatan atrasos en la ejecución del calendario agrícola, debido a la mala condición alimenticia y de salud de los bueyes al inicio de la campaña. Para remediar esto, el proyecto URDOC estableció y validó un conjunto de medidas que permiten el mejoramiento del estado de los animales de tiro. La difusión se hace en el marco del enfoque "consejo a la explotación familiar". La continuidad del impacto positivo de la tracción animal dependerá del compromiso de los diferentes actores en cuanto al refuerzo de los servicios de apoyo: consejo, crédito, mantenimiento/fabricación del material y servicios veterinarios.

Palabras clave: Energía animal - Política agrícola - Delta - Río Niger - Malí. 